\title{
Hierarchical Reactivity of Enzyme-Mediated Phosphorus Recycling from Organic Mixtures by Aspergillus niger Phytase
}

\author{
Mina Solhtalab ${ }^{1}$, Annaleise R. Klein ${ }^{1,2,3}$, Ludmilla Aristilde ${ }^{1,2, *}$ \\ ${ }^{1}$ Department of Biological and Environmental Engineering, College of Agriculture and Life \\ Sciences, Cornell University, Ithaca, NY 14853, USA \\ ${ }^{2}$ Department of Civil and Environmental Engineering, McCormick School of Engineering and \\ Applied Science, Northwestern University, Evanston, Il 60208, USA \\ ${ }^{3}$ Current address: Australian Synchrotron, Australian Nuclear Science and Technology \\ Organisation, Clayton, VIC 3168 Australia
}

*Corresponding Author: ludmilla.aristilde@northwestern.edu; Phone: 847-491-2999

\section{SUPPLEMENTARY INFORMATION}

Appendix A: Details on materials

Appendix B: Details on Chromatographic Procedure and Inorganic P Analysis

Appendix C: Governing Equations of Kinetic Models of Enzymatic Hydrolysis

Appendix D: Single-Substrate Experimental Data and Kinetic Modeling

Appendix E: Mixed-Substrate Experimental Data and Kinetic Modeling

Appendix F: The Transformation Coefficients of $P_{0}$ Compounds

Appendix G: The Initial Rates of Dephosphorylation at Different Concentrations of ATP and G6P

Appendix H: Statistical Analysis of Initial Rate differences in Mixed- and Single Substrate Reactions for ATP and G6P

Appendix I: Standard Deviation Calculation for the Ratios of Initial Rates Based on the Taylor Series Method

APPENDIX J: Interaction Criteria in Molecular Modeling Simulations 


\section{APPENDIX A: Details on Materials}

Table S1. Details of the venders for the chemical used in this study. All phosphorylated compounds were of $\geq 89 \%$ purity.

\begin{tabular}{|c|c|}
\hline Chemicals name & Vendor \\
\hline Phytic acid sodium salt hydrate & Sigma, MO, USA \\
\hline D-Glucose-6-phosphate sodium salt & Sigma, MO, USA \\
\hline D-Ribose-5-phosphate disodium salt dihydrate & Sigma, MO, USA \\
\hline D-Fructose 1,6-bisphosphate & Sigma, MO, USA \\
\hline Adenosine 5'-triphosphate disodium salt hydrate & Sigma, MO, USA \\
\hline Adenosine 5'-monophosphate monohydrate & Sigma, MO, USA \\
\hline D-(-)-Ribose & Sigma, MO, USA \\
\hline Adenosine 5'-diphosphate disodium salt dihydrate & Chem Impex Int'l Inc., IL, USA \\
\hline Adenosine free base & Chem Impex Int'l Inc., IL, USA \\
\hline D-Fructose & Fisher Scientific, NH, USA \\
\hline D-(+)-Glucose anhydrous & MP Biomedicals, OH, USA \\
\hline D-myoinositol-1-phosphate & Cayman Chemical Company, MI, USA \\
\hline D-myoinositol-1,4-diphosphate & Cayman Chemical Company, MI, USA \\
\hline D-myoinositol-1,3,4-triphosphate & Cayman Chemical Company, MI, USA \\
\hline D-myoinositol-1,3,4,5-tetraphosphate & Cayman Chemical Company, MI, USA \\
\hline D-myoinositol-1,3,4,5,6-pentaphosphate & Cayman Chemical Company, MI, USA \\
\hline D-Fructose 6-phosphate & EMD Millipore Corp, MA, USA \\
\hline Phytase from Aspergillus niger (Natuphos) & Gift from BASF, NJ, USA \\
\hline
\end{tabular}


APPENDIX B: Details on Chromatographic Procedure and Inorganic P Analysis

Details on chromatographic procedure:

Using Solvent A composed of $100 \%$ methanol and solvent B composed of a $97: 3$ mixture of water:methanol with acetic acid $(15 \mathrm{mM})$ and the ion-pairing agent tributylamine $(10 \mathrm{mM})$, the LC separation (using a $2.1 \times 100 \mathrm{~mm} \times 1.7 \mu \mathrm{m}$ ACQUITY UPLC BEH C18 column; Waters, Milford, MA) was achieved by the following multi-step gradient for solvent B: 4 mins preequilibration; 0\%, $0 \mathrm{~min}, 0 \%$; $1 \mathrm{~min}, 30 \%$; $2.5 \mathrm{~min}, 60 \%$; $9 \mathrm{~min}, 75 \%$; $9.5 \mathrm{~min}, 95 \%$. To separate G6P and F6P in these samples, a gradient of 95:5 methanol:water was used for 60 mins.

\section{Details of $\mathrm{P}_{\mathrm{i}}$ analysis:}

Samples were neutralized with $\mathrm{NaOH}$ and diluted with Milli-Q water to obtain $\mathrm{Pi}$ concentration in the range of the calibration range $(2.5 \mu \mathrm{M}-40 \mu \mathrm{M})$ prepared with potassium phosphate $\left(\mathrm{K}_{2} \mathrm{HPO}_{4}\right)$. We added $400 \mu \mathrm{L}$ ammonium molybdate reagent to $10 \mathrm{~mL}$ of the prepared samples followed by addition of one drop of $\mathrm{SnCl}_{2}$. The solutions were well mixed and, after 11 mins at room temperature, the absorbance was read at $690 \mathrm{~nm}$ with UV-Vis spectrometer. 


\section{APPENDIX C: Governing Equations of Kinetic Models of Enzymatic Hydrolysis}

The governing equations and analytical solutions for the sequential dephosphorylation of ATP (adenosine triphosphate) as a single substrate into its by-products ADP (adenosine diphosphate) and AMP (adenosine monophosphate), and $\mathrm{P}_{\mathrm{i}}$ (inorganic phosphate):

$$
\begin{aligned}
& \frac{d[\mathrm{ATP}]}{d t}=-k_{A T P}[\mathrm{ATP}] \\
& {[\mathrm{ATP}]=[\mathrm{ATP}]_{0} \exp \left(-k_{\mathrm{ATP}} t\right)} \\
& \frac{d[\mathrm{ADP}]}{d t}=k_{A T P}[\mathrm{ATP}]-k_{A D P}[\mathrm{ADP}] \\
& {[\mathrm{ADP}]=\frac{k_{A T P}[\mathrm{ATP}]_{0}}{k_{\mathrm{ADP}}-k_{\mathrm{ATP}}} \exp \left(-k_{\mathrm{ATP}} t\right)+\left([\mathrm{ADP}]_{0}-\frac{k_{\mathrm{ATP}}[\mathrm{ATP}]_{0}}{k_{\mathrm{ADP}}-k_{\mathrm{ATP}}}\right) \exp \left(-k_{\mathrm{ADP}} t\right)} \\
& \frac{d[\mathrm{AMP}]}{d t}=k_{A D P}[\mathrm{ADP}]-k_{A M P}[\mathrm{AMP}] \\
& {[\mathrm{AMP}]=\frac{k_{\mathrm{ADP}} k_{\mathrm{ATP}}[\mathrm{ATP}]_{0}}{\left(k_{A D P}-k_{A T P}\right)\left(k_{A M P}-k_{A T P}\right)} \exp \left(-k_{\mathrm{ATP}} t\right)+} \\
& \frac{k_{\mathrm{ADP}}[\mathrm{ADP}]_{0}\left(k_{\mathrm{ADP}}-k_{\mathrm{ATP}}\right)-k_{\mathrm{ADP}} k_{\mathrm{ATP}}[\mathrm{ATP}]_{0}}{\left(k_{\mathrm{ADP}}-k_{\mathrm{ATP}}\right)\left(k_{\mathrm{AMP}}-k_{\mathrm{ADP}}\right)} \exp \left(-k_{\mathrm{ADP}} t\right)+ \\
& \quad\left(k_{\mathrm{AMP}}-k_{\mathrm{ATP}}\right)\left(k_{\mathrm{AMP}}-k_{\mathrm{ADP}}\right) \\
& \frac{d[\mathrm{Pi}]}{d t}=k_{A T P}[\mathrm{ATP}]+k_{A D P}[\mathrm{ADP}]+k_{A M P}[\mathrm{AMP}] \\
& {[P i]=[A T P]_{0}\left(1-\exp \left(-k_{A T P} t\right)\right)+[A D P]_{0}\left(1-\exp \left(-k_{A D P} t\right)\right)+[A M P]_{0}\left(1-\exp \left(-k_{A M P} t\right)\right)}
\end{aligned}
$$

The governing equations for the sequential dephosphorylation of ADP as a single substrate into its by-products $A M P$, and $\mathrm{P}_{\mathrm{i}}$ :

$$
\begin{aligned}
& \frac{d[\mathrm{ADP}]}{d t}=-k_{\mathrm{ADP}}[\mathrm{ADP}] \\
& \quad[\mathrm{ADP}]=[\mathrm{ADP}]_{0} \exp \left(-k_{\mathrm{ADP}} t\right) \\
& \frac{d[\mathrm{AMP}]}{d t}=k_{\mathrm{ADP}}[\mathrm{ADP}]-k_{\mathrm{AMP}}[\mathrm{AMP}] \\
& \quad[\mathrm{AMP}]=\frac{k_{\mathrm{ADP}}[\mathrm{ADP}]_{0}}{k_{\mathrm{AMP}}-k_{\mathrm{ADP}}} \exp \left(-k_{\mathrm{ADP}} t\right)+\left([\mathrm{AMP}]_{0}-\frac{k_{\mathrm{ADP}}[\mathrm{ADP}]_{0}}{k_{\mathrm{AMP}}-k_{\mathrm{ADP}}}\right) \exp \left(-k_{\mathrm{AMP}} t\right) \\
& \frac{d[\mathrm{~A}]}{d t}=k_{\mathrm{AMP}}[\mathrm{AMP}]-k_{A}[A]
\end{aligned}
$$




$$
\begin{gathered}
{[\text { Adenosine }]=\frac{k_{\mathrm{AMP}} k_{\mathrm{ADP}}[\mathrm{ADP}]_{0}}{\left(k_{A M P}-k_{A D P}\right)\left(k_{A}-k_{A D P}\right)} \exp \left(-k_{\mathrm{ADP}} t\right)+} \\
\frac{k_{\mathrm{AMP}}[\mathrm{AMP}]_{0}\left(k_{\mathrm{AMP}}-k_{\mathrm{ADP}}\right)-k_{\mathrm{AMP}} k_{\mathrm{ADP}}[\mathrm{ADP}]_{0}}{\left(k_{\mathrm{AMP}}-k_{\mathrm{ADP}}\right)\left(k_{\mathrm{A}}-k_{\mathrm{AMP}}\right)} \exp \left(-k_{\mathrm{AMP}} t\right)+ \\
\frac{k_{\mathrm{AMP}}[\mathrm{AMP}]_{0}\left(k_{\mathrm{A}}-k_{\mathrm{ADP}}\right)-k_{\mathrm{AMP}} k_{\mathrm{ADP}}[\mathrm{ADP}]_{0}}{\left(k_{\mathrm{A}}-k_{\mathrm{ADP}}\right)\left(k_{\mathrm{A}}-k_{\mathrm{AMP}}\right)} \exp \left(-k_{\mathrm{A}} t\right) \\
\frac{d[\mathrm{Pi}]}{d t}=k_{A D P}[\mathrm{ADP}]+k_{A M P}[\mathrm{AMP}] \\
{[P i]=[A D P]_{0}\left(1-\exp \left(-k_{A D P} t\right)\right)+[A M P]_{0}\left(1-\exp \left(-k_{A M P} t\right)\right)}
\end{gathered}
$$

The governing equations for FBP, F6P, and $\mathrm{P}_{\mathrm{i}}$ from the sequential dephosphorylation steps are:

$$
\begin{aligned}
& \frac{d[\mathrm{FBP}]}{d t}=-k_{\mathrm{FBP}}[\mathrm{FBP}] \\
& {[\mathrm{FBP}]=[\mathrm{FBP}]_{0} \exp \left(-k_{\mathrm{FBP}} t\right)} \\
& \frac{d[\mathrm{~F} 6 \mathrm{P}]}{d t}=k_{\mathrm{FBP}}[\mathrm{FBP}]-k_{\mathrm{F} 6 \mathrm{P}}[\mathrm{F} 6 \mathrm{P}] \\
& {[\mathrm{F} 6 \mathrm{P}]=\frac{k_{\mathrm{FBP}}[\mathrm{FBP}]_{0}}{k_{\mathrm{F} 6 \mathrm{P}}-k_{\mathrm{FBP}}} \exp \left(-k_{\mathrm{FBP}} t\right)+\left([\mathrm{F} 6 \mathrm{P}]_{0}-\frac{k_{\mathrm{FBP}}[\mathrm{FBP}]_{0}}{k_{\mathrm{F} 6 \mathrm{P}}-k_{\mathrm{FBP}}}\right) \exp \left(-k_{\mathrm{F} 6 \mathrm{P}} t\right)} \\
& \frac{d[\mathrm{Frc}]}{d t}=k_{\mathrm{F} 6 \mathrm{P}}[\mathrm{F} 6 \mathrm{P}] \\
& {[\mathrm{Fructose}]=\frac{k_{\mathrm{F} 6 \mathrm{P}}[\mathrm{FBP}]_{0}}{k_{\mathrm{F} 6 \mathrm{P}}-k_{\mathrm{FBP}}}\left(1-\exp \left(-k_{\mathrm{FBP}} t\right)\right)+\frac{k_{\mathrm{FBP}}[\mathrm{FBP}]_{0}}{k_{\mathrm{F} 6 \mathrm{P}}-k_{\mathrm{FBP}}}\left(1-\exp \left(-k_{\mathrm{F} 6 \mathrm{P}} t\right)\right)} \\
& \frac{d[\mathrm{Pi}]}{d t}=k_{F B P}[\mathrm{FBP}]+k_{F 6 P}[\mathrm{~F} 6 \mathrm{P}] \\
& {[\mathrm{Pi}]=[F B P]_{0}\left(1-\exp \left(-k_{\mathrm{FBP}} t\right)\right)+[F 6 P]_{0}\left(1-\exp \left(-k_{\mathrm{F} 6 \mathrm{P}} t\right)\right)} \\
& { }(1)
\end{aligned}
$$

The following equations was used for modeling the concentrations of sugar phosphates (G6P, F6P and R5P), their sugar biproducts (Glucose, Fructose and Ribose) and released $\mathrm{P}_{\mathrm{i}}$ in singlesubstrate reactions:

$$
\frac{d[\mathrm{XP}]}{d t}=-k_{\mathrm{XP}}[\mathrm{XP}]
$$


$[\mathrm{XP}]=[\mathrm{XP}]_{0} \exp \left(-k_{\mathrm{XP}} t\right)$

$\frac{d[\mathrm{X}]}{d t}=-k_{\mathrm{XP}}[\mathrm{XP}]$

$[\mathrm{X}]=[\mathrm{XP}]_{0}\left(1-\exp \left(-k_{\mathrm{XP}} t\right)\right.$

$\frac{d\left[\mathrm{P}_{i}\right]}{d t}=-k_{\mathrm{XP}}[\mathrm{XP}]$

$\left[P_{i}\right]=[\mathrm{XP}]_{0}\left(1-\exp \left(-k_{\mathrm{XP}} t\right)\right.$

To model the concentrations of species in the mixed-substrate reactions, the following equations were used: Eqs. [2], [4] and [6] for ATP, ADP and AMP, respectively; Eqs. [17], [19] and [21] for FBP, F6P and fructose, respectively; Eq. [26] for G6P and R5P, and Eq. [28] for Glucose. During preliminary experiments, we found that the PhyA extract can mediate adenosine hydrolysis to ribose (Appendix $\mathrm{D}, \mathrm{SI}$ ). Therefore, to model the rate of ribose production from the mixture, we considered both R5P dephosphorylation and hydrolysis of adenosine produced from ATP dephosphorylation as follows:

$\frac{d[\text { Ribose }]}{d t}=k_{\mathrm{R} 5 \mathrm{P}}[\mathrm{R} 5 \mathrm{P}]+k_{\text {adenosine }}[$ adenosine $]$

$[$ Ribose $]=[\mathrm{R} 5 \mathrm{P}]_{0}\left(1-\exp \left(-k_{R 5 P} t\right)+[\text { adenosine }]_{0}\left(1-\exp \left(-k_{\text {adenosine }} t\right)\right.\right.$ 


\section{APPENDIX D: Single-Substrate Experimental Data and Kinetic Modeling}

We studied the hydrolysis patterns of phytate, adenosine-based ribonucleotides (AXP) and sugar phosphates via phytase A. Due to addition of $\mathrm{HCl}$ for stopping the reaction, ATP and ADP were partially degraded to the lower phosphorylated forms. Therefore, their initial concentrations were lower than $400 \mu \mathrm{M}$ (Figure S1 A and D).

$m I P_{6}$ and By-products. We tracked the kinetic evolution of $\mathrm{mIP}$ by-products from the sequential dephosphorylation of $\mathrm{mIP}_{6}$ at two different scenarios (Figure $\mathrm{S} 1$ ). In the first scenario with high-substrate concentration $(2 \mathrm{mM} \mathrm{mIP})$ and relatively low enzyme concentration $(0.15 \mathrm{U} / \mathrm{mL}$ PhyA) (Figure $\mathrm{S} 1 \mathrm{~A}), \mathrm{mIP}_{6}$ was fully converted to $\mathrm{mIP}_{4}$ by 5 mins where $\mathrm{mIP}_{5}$ was a short-lived intermediate (Figure S1 A), similar to previous observations ${ }^{1-}$

3. The $\mathrm{mIP}_{4}$ product reached a maximum $(2.12 \pm 0.07 \mathrm{mM})$ at 5 mins and dropped to 0.79 $\mathrm{mM}$ by 10 mins, at which point appreciable level of $\mathrm{mIP}_{3}(1.05 \pm 0.02 \mathrm{mM})$ was detected (Figure S1 A). Disappearance of $\mathrm{mIP}_{3}$ and the maximum concentration of mIP2 (1.28 \pm 0.03 $\mathrm{mM}$ ) both occurred at $1 \mathrm{~h}$ (Figure $\mathrm{S} 1 \mathrm{~A}$ ). For the next five hours, $\mathrm{mIP}_{2}$ was slowly converted to $\mathrm{mIP}_{1}$ (Figure $2 \mathrm{~A}$ ). For the remaining duration of the experiment (i.e. $48 \mathrm{~h}$ total), only $30 \%$ of the millimolar concentration of $\mathrm{mIP}_{1}$ was dephosphorylated (Figure S1A). Due to the large amount of $\mathrm{P}_{i}$ in solution, which represented over $90 \%$ of total $\mathrm{P}$, the lack of complete dephosphorylation of mIP1 may be due to enzyme inhibition by $\mathrm{P}_{\mathrm{i}}$ as previously proposed (Figure S1 A) ${ }^{4,5}$. In a different experimental scenario with 20 times higher enzyme concentration ( $3 \mathrm{U} / \mathrm{mL}$ PhyA) and 5 times lower mIP6 concentration (400 $\mu \mathrm{M}$ mIP6), we obtained fast and complete disappearance of mIP6 by $30 \mathrm{~s}$ and no mIP5, mIP4, mIP3, or mIP1 was detected in solution (Figure S1 B). Within $1 \mathrm{~h}$, the total $\mathrm{P}_{\mathrm{i}}$ concentration $(1.76 \pm$ $0.02 \mathrm{mM}$ ) reflected the near-equivalent $P_{i}$ from the complete dephosphorylation of mIP6 (Figure S1 B). In previous studies of mIP6 dephosphorylation by PhyA, different final solution by-products were reported: only mIP4 and mIP3, ${ }^{2}$ only mIP1, ${ }^{6}$ or only mIP2 and 
$\mathrm{mIP}^{7}$. Based on our results, the inconsistencies in these previous reports ${ }^{2,6,7}$ are likely due to amount of PhyA with respect to the mIP6 and $P_{i}$ concentrations, which would determine to what extent complete versus partial sequential dephosphorylation of mIP6 will be achieved.

ATP. In an ATP-only solution (initial concentration of $272 \mu \mathrm{M}$ ), all ATP was consumed abruptly by enzyme phytase A (Figure S2 A). A very negligible amount of ATP $(7.69 \mu \mathrm{M})$ was measured after 30 seconds and then it went down to zero. As the product of this hydrolysis, ADP concentration grew strikingly to about $252 \mu \mathrm{M}$ after only 30 seconds (Figure S2 A). The exponential consumption of ADP and production of AMP started immediately. ADP concentration dropped down to almost zero after 6 hours and AMP reached its peak (205 $\mu M)$ in one hour (Figure S2 B). Phytase A was also able to dephosphorylate all AMP and produce Adenosine. Adenosine rose to its highest concentration at 6 hour-time point (Figure S1 C). As we used a crude sample of enzyme, we also saw the removal of Adenosine, which could be due to the presence of other enzymes in the crude extract. Therefore, the crude extract of phytase A provided ribose $(429 \mu \mathrm{M})$ (Figure S3) as the final product of ribonucleotide degradations. After 48 hours of incubation of ATP solution with phytase A, about $948 \mu M$ of Pi was released (Figure S2 C).

ADP. Phytase activity against ADP was measured in an ADP-only solution. About half of the ADP present in the sample was used rapidly during the first 5 minutes (Figure S2 D) and the rest was consumed entirely by the first one hour of the incubation. AMP production also peaked in about 5 minutes $(\sim 269 \mu \mathrm{M})$ (Figure S2D and E). After 24 hours, almost no AMP was observed in the reaction mixture (Figure S2 F). The production of Adenosine started after one hour and the highest concentration $(\sim 130 \mu \mathrm{M})$ was observed at 6 hours (Figure S1 E and F). A total of $703 \mu \mathrm{M}$ Pi was produced after 48 hours of incubation of pure $400 \mu \mathrm{M}$ ADP with phytase A (Figure S2 F). 
AMP. Phytase started using AMP after 20 minutes of incubation. AMP concentration declined gently (Figure S2 G) followed by adenosine production that got to its highest level in 6 hours (Figure S2 $\mathrm{H}$ and I). The total Pi production stabilized to $437 \mu \mathrm{M}$ after 48 hours (Figure S2 I). The kinetic constants calculated from analytical models (Figure 4) revealed that the consumption-rate of AMP ( $k_{\text {trans }}=0.0043 \pm 0.0005$ st.er.) is much lower than ADP $\left(k_{\text {trans }}=0.1357 \pm 0.0271\right.$ st.er.) (Figure 4$)$ and both have a huge difference with the rate of enzymatic hydrolysis of ATP ( $k_{\text {trans }}=3.4637 \pm 0.1960$ st.er.) (Figure 4$)$ in single substrate solutions. This suggests that PhyA enzyme greatly preferred the higher phosphorylated ribonucleotides.

A striking difference between PhyA activity towards mIP6 versus AXP was reported in previous studies ${ }^{3,8-10}$. Considering this huge difference, the activities against ATP, ADP or AMP were not very different, though, a comparison between the reported rates does not give a consistent hierarchy across the literature ${ }^{3,8-10}$.

Fructose-1,6-bis phosphate. The PhyA extract had a high activity towards FBP in a similar fashion as the other multi-phosphorylated substrates like ATP and ADP. FBP was used up in less than 30 seconds of incubation time with PhyA (Figure S4 A). From 30 seconds on, a combination of F6P/F1P concentration which had surged to about $359 \mu \mathrm{M}$ by 30 seconds (Figure S4 A) started getting dephosphorylated to fructose with a gentle slope (Figure S4 A). All F6P was dephosphorylated by 48 hours (Figure S4 A). A maximum of $224 \mu \mathrm{M}$ of Fructose and a total of $615 \mu \mathrm{MPi}$ was released from enzymatic dephosphorylation of FBP after 48 hours (Figure S4 A).

Fructose 6-phosphate. Slow dephosphorylation of F6P started from the first 30 second of incubation (Figure S4 B). Subsequently, F6P concentration decreased to half of its initial value $(216 \mu \mathrm{M})$ by 6 hours, and it was all dephosphorylated to fructose in 48 hours (Figure S4 B). A total of $390 \mu \mathrm{M}$ Pi was released from $416 \mu \mathrm{M}$ F6P-initial concentration (Figure S5 B). 
Glucose-6-phosphate. The dephosphorylation trend for G6P in single-substrate reaction (Figure S4 C) was very similar to F6P (Figure S4 B). G6P concentration was halved after 6 hours and it was all dephosphorylated by 48 hours (Figure S4 C). About $315 \mu \mathrm{M}$ Pi was released from $329 \mu \mathrm{M}$ initial G6P (Figure S4 C).

Ribose-5-phosphate. PhyA dephosphorylated R5P more sharply compared to G6P and F6P (Figure S4 D, C, B). When incubated with phytase A, the half-life of R5P was about 2.5 hours (Figure S4 D). Almost all R5P was dephosphorylated after 24 hours (Figure S4 D). The total Pi released from R5P was $391 \mu \mathrm{M}$ (Figure S4 D).
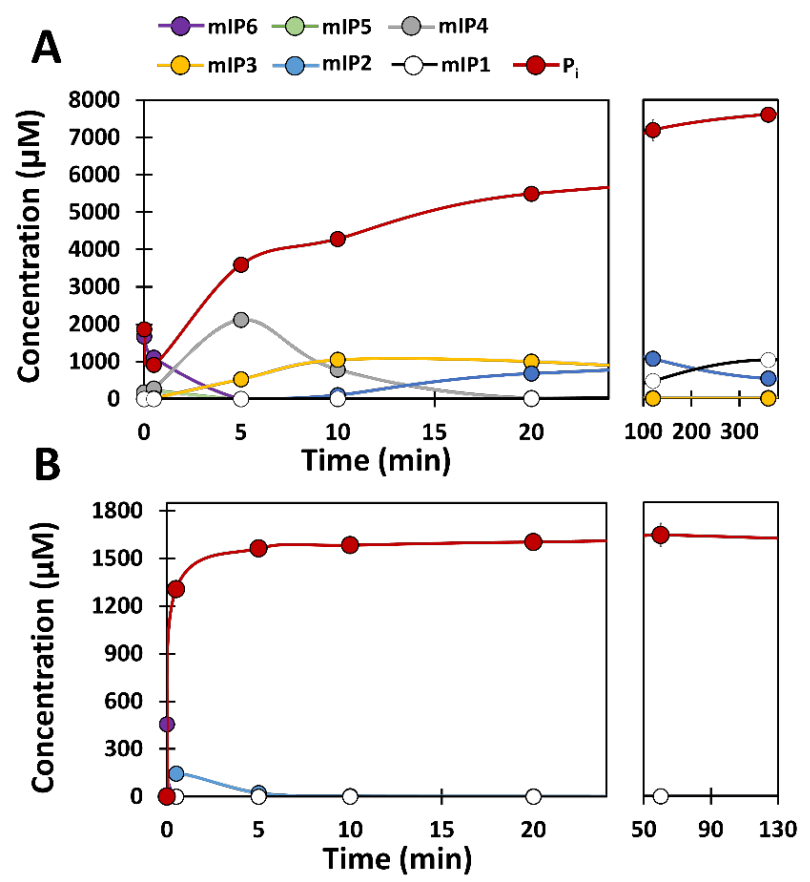

Figure S1. Sequential dephosphorylation of mIP6 and by-products at two different concentrations of mIP6 and PhyA: (A) 2 mM mIP6 with $0.15 \mathrm{U} / \mathrm{ml}$ PhyA or (B) $400 \mu \mathrm{M}$ mIP6 with $3 \mathrm{U} / \mathrm{ml}$ PhyA. Lines are added as a guide for the eye. The full kinetic data obtained over $48 \mathrm{~h}$ (or 2,880 mins) are shown in Appendix D (SI). 

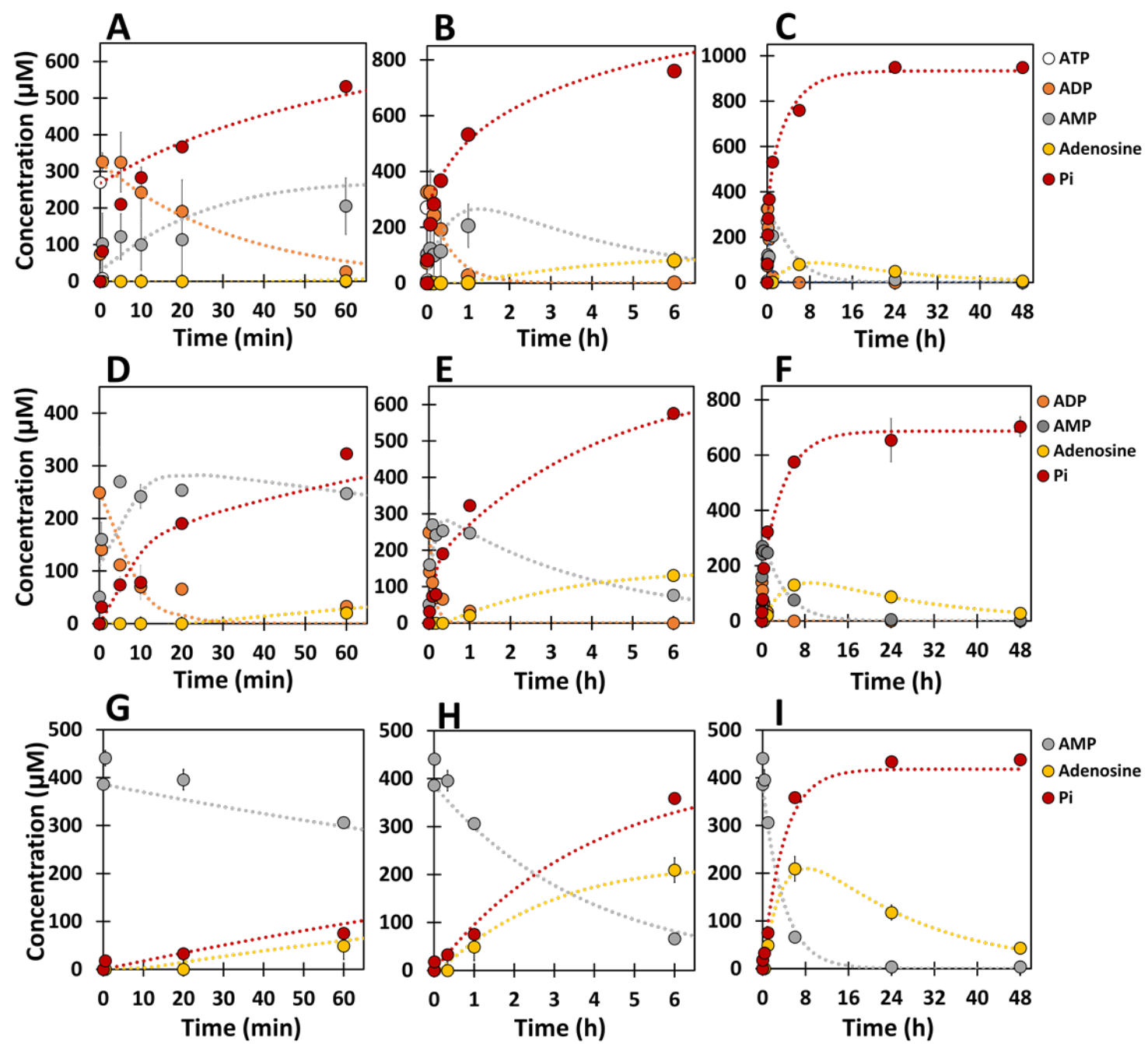

Figure S2. Dephosphorylation patterns of ribonucleotides by PhyA extract. The stepwise PhyAmediated degradation of adenosine-based ribonucleotides including ATP (A, B, C), ADP (D, E, F) and $\operatorname{AMP}(\mathrm{G}, \mathrm{H}, \mathrm{I})$ and their byproducts are shown in three timespans for single-substrate reaction solutions. The models of the reaction rates are graphed as dotted lines. 


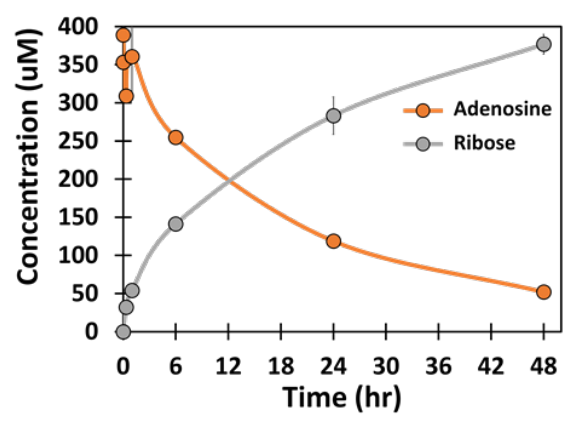

Figure S3. Degradation of adenosine to ribose by the activity of the PhyA extract.
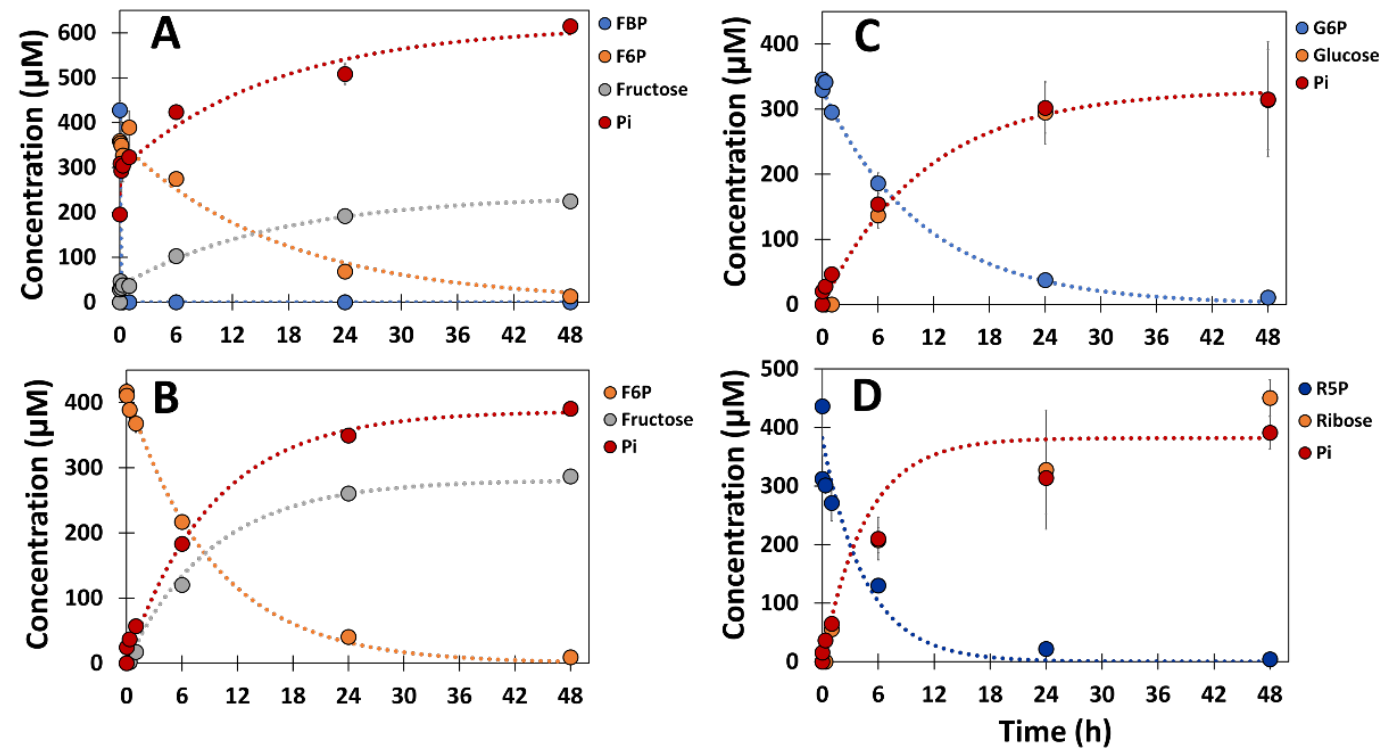

Figure S4. Dephosphorylation patterns of sugar phosphates by crude phytase A. Dephosphorylation trends of sugar phosphates are shown for FBP (A), F6P (B), G6P (C) and R5P (D) in single-substrate reactions. The models of the reaction rates are graphed as dotted lines. Note, the $\mathrm{Pi}$ and sugar models in $\mathrm{C}$ and $\mathrm{D}$ are overlapped. 
APPENDIX E: Mixed-Substrate Experimental Data and Kinetic Modeling
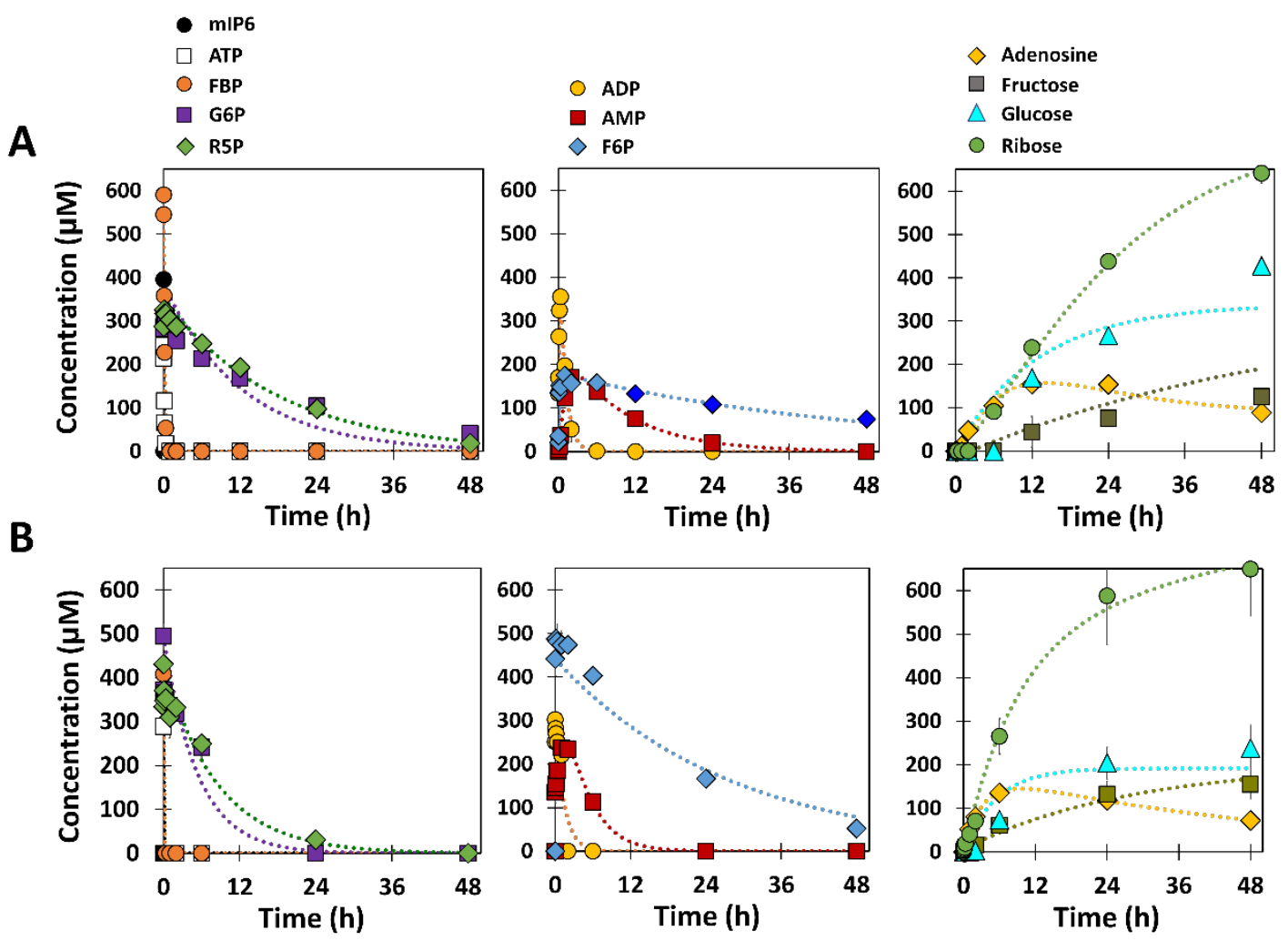

Figure S5. Full dephosphorylation kinetics of a mixture of $P_{0}$ compounds by PhyA extract with $(A)$ or without (B) the indigenous substrate (mIP6) of enzyme. Left panels show the dephosphorylation kinetics of compounds in the starting mixture: ATP (white squares), FBP (orange circles), G6P (purple squares), and R5P (green diamonds), with/without mIP6 (black circles). Middle panels and right panels show, respectively, the kinetics of monophosphorylated and unphosphorylated daughter products. 


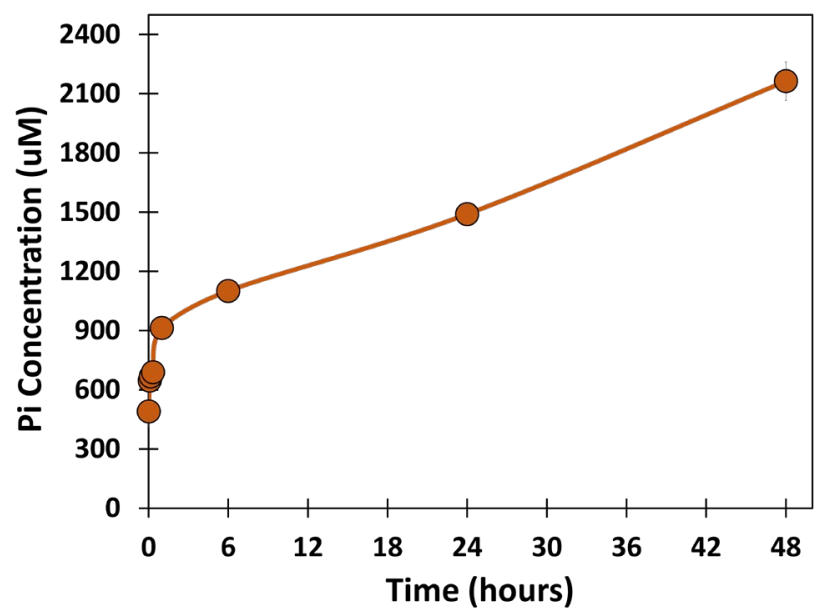

Figure S6. Total $P_{i}$ released during dephosphorylation of $P_{0}$ mixture without mIP6. 


\section{APPENDIX F: The Transformation Coefficients of $P_{0}$ Compounds}

Table S2. The transformation coefficients of $\mathrm{P}_{\mathrm{o}}$ compounds in single substrate solution and mixtures with or without $\mathrm{mIP}_{6}$.

\begin{tabular}{|clll|}
\hline & \multicolumn{3}{c|}{$\mathrm{K}_{\text {decay }}\left(\mathrm{h}^{-1}\right) \pm$ Standard error } \\
\cline { 2 - 4 } $\begin{array}{c}\text { Po } \\
\text { Compound }\end{array}$ & Single substrate & Mixture without mIP6 & Mixture with mIP6 \\
FBP & $205 \pm 10$ & $205 \pm 10$ & $8.09 \pm 4.92 \times 10^{-1}$ \\
ATP & $205 \pm 12$ & $205 \pm 10$ & $6.03 \pm 1.08$ \\
ADP & $8.14 \pm 1.63$ & $6.96 \times 10^{-1} \pm 9.00 \times 10^{-2}$ & $1.03 \pm 1.08 \times 10^{-1}$ \\
AMP & $2.58 \times 10^{-1} \pm 3.00 \times 10^{-2}$ & $2.82 \times 10^{-1} \pm 3.00 \times 10^{-2}$ & $1.02 \times 10^{-1} \pm 1.98 \times 10^{-3}$ \\
G6P & $9.00 \times 10^{-2} \pm 5.46 \times 10^{-3}$ & $1.86 \times 10^{-1} \pm 4.20 \times 10^{-2}$ & $7.80 \times 10^{-2} \pm 1.20 \times 10^{-2}$ \\
R5P & $2.16 \times 10^{-1} \pm 3.60 \times 10^{-2}$ & $1.20 \times 10^{-1} \pm 1.80 \times 10^{-2}$ & $5.40 \times 10^{-2} \pm 6.00 \times 10^{-3}$ \\
F6P & $1.08 \times 10^{-1} \pm 5.10 \times 10^{-3}$ & $3.60 \times 10^{-2} \pm 4.32 \times 10^{-3}$ & $1.80 \times 10^{-2} \pm 6.00 \times 10^{-3}$ \\
\hline
\end{tabular}


APPENDIX G: The Initial Rates of Dephosphorylation at Different Concentrations of ATP and G6P

Table S3. The initial rates of dephosphorylation at different concentrations of ATP and G6P in single substrate solution and mixture.

\begin{tabular}{|c|c|c|c|c|c|c|c|}
\hline \multicolumn{4}{|c|}{ ATP } & \multicolumn{4}{|c|}{ G6P } \\
\hline \multicolumn{2}{|c|}{ Single substrate } & \multicolumn{2}{|c|}{ Mixture } & \multicolumn{2}{|c|}{ Single substrate } & \multicolumn{2}{|c|}{ Mixture } \\
\hline [ATP-P] $\mathrm{mM}$ & $\mathrm{V}_{\text {ATP }}\left(h^{-1}\right) \pm$ s.d. ${ }^{*}$ & [fractional ATP-P] $_{\text {Mix }}$ & $\mathrm{V}_{\mathrm{ATP}}\left(\mathrm{h}^{-1}\right) \pm \mathrm{s.d}$ & [G6P-P] mM & $V_{G G P}\left(h^{-1}\right) \pm$ s.d. & [fractional G6P-P] $]_{\text {Mix }}$ & $\mathrm{V}_{\mathrm{GGP}}\left(\mathrm{h}^{-1}\right) \pm$ s.d. \\
\hline $10^{-1}$ & $49.8 \pm 3.1$ & $8.57 \times 10^{-2}$ & $6.70 \pm 1.10$ & $10^{-1}$ & $7.89 \times 10^{-2} \pm 1.26 \times 10^{-2}$ & $2.44 \times 10^{-2}$ & ------ \\
\hline $10^{0}$ & $126 \pm 2$ & $4.84 \times 10^{-1}$ & $32.7 \pm 9.0$ & $10^{0}$ & $8.96 \times 10^{-1} \pm 2.27 \times 10^{-1}$ & $2.00 \times 10^{-1}$ & $7.83 \times 10^{-1} \pm 7.35 \times 10^{-2}$ \\
\hline 10 & $301 \pm 47$ & $9.04 \times 10^{-1}$ & $137 \pm 44$ & 10 & $10.7 \pm 2.4$ & $7.14 \times 10^{-1}$ & $10.3 \pm 1.7$ \\
\hline
\end{tabular}

* s.d. is the abbreviation for standard deviation. 
APPENDIX H: Statistical Analysis of Initial Rate differences in Mixed- and Single Substrate Reactions for ATP and G6P

Table S4. Details on the p-values from unpaired t-test to show the statistical significances between mixed-and single-substrate reactions

\begin{tabular}{|c|c|c|c|c|c|c|}
\hline & \multicolumn{3}{|c|}{ ATP } & \multicolumn{3}{|c|}{ G6P } \\
\hline & $0.1 \mathrm{mM}$ & $1 \mathrm{mM}$ & $10 \mathrm{mM}$ & $0.1 \mathrm{mM}$ & $1 \mathrm{mM}$ & $10 \mathrm{mM}$ \\
\hline P-value & $<0.001$ & $<0.001$ & $<0.05$ & ---- & 0.460 & 0.842 \\
\hline Significance & $* * *$ & $* * *$ & $*$ & ---- & ns & ns \\
\hline $\begin{array}{l}\text { Averaged Mixed-substrate rate } \\
\left(\text { avg } \pm \text { s.d. } h^{-1} \text { ) }\right.\end{array}$ & $6.70 \pm 1.10$ & $32.7 \pm 9.0$ & $137 \pm 44$ & ---- & $7.83 \times 10^{-1} \pm 7.35 \times 10^{-2}$ & $10.3 \pm 1.7$ \\
\hline $\begin{array}{l}\text { Single-substrate rate } \\
\left(\text { avg } \pm \text { s.d. } h^{-1}\right)\end{array}$ & $49.8 \pm 3.1$ & $126 \pm 2$ & $301 \pm 47$ & $7.89 \times 10^{-2} \pm 1.26 \times 10^{-2}$ & $8.96 \times 10^{-1} \pm 2.27 \times 10^{-1}$ & $10.7 \pm 2.4$ \\
\hline
\end{tabular}

*** extremely statistically significant

** $\quad$ very statistically significant

* $\quad$ statistically significant

ns not statistically significant 
APPENDIX I: Standard Deviation Calculation for the Ratios of Initial Rates Based on the Taylor Series Method

In order to calculate the standard deviations of the ratios of initial rates of dephosphorylation of ATP and G6P in mixture versus single substrate scenarios, first the standard deviations of the triplicate initial rates were calculated. Then, based on the following equation, which is the solution of Taylor series ${ }^{11}$, the variance of the ratios was calculated:

$$
\operatorname{varience}\left(\frac{M}{s}\right)=\frac{\left(\mu_{M}\right)^{2}}{\left(\mu_{s}\right)^{2}}\left[\frac{\sigma_{M}^{2}}{\left(\mu_{M}\right)^{2}}-2 \frac{\operatorname{Cov}(M, S)}{\mu_{R} \cdot \mu_{s}}+\frac{\sigma_{s}^{2}}{\left(\mu_{s}\right)^{2}}\right]
$$

Where $\mu$ represents the average value of triplicate initial rates, $\sigma$ represents the standard deviation of triplicate initial rates, $M$ represents the mixed-substrate condition, $S$ represents the single-substrate condition and Cov represents the covariance for dependent data sets, which is zero for independent sets of data, like our data. Subsequently, the standard deviations of the ratios were calculated by taking the square root of the corresponding variances. 
APPENDIX J: Interaction Criteria in Molecular Modeling Simulations

Table S5. Details on the criteria for calculations of electrostatic interactions

\begin{tabular}{|l|l|l|}
\hline Interaction type & $\begin{array}{l}\text { Maximum Distance } \\
(\AA)\end{array}$ & $\begin{array}{l}\text { Maximum Angle } \\
\left({ }^{\circ}\right)\end{array}$ \\
\hline Charge-Charge & 5.6 & - \\
\hline$\pi-C a t i o n$ & 5 & 40 \\
\hline$\pi-$ Anion & 5 & 40 \\
\hline$\pi-\pi$ Centroid & 6 & 50 \\
\hline
\end{tabular}




\section{References}

1. Greiner, R.; Konietzny, U.; Jany, K. D. Purification and Characterization of Two Phytases from Escherichia Coli. Archives of Biochemistry and Biophysics 1993, 303 (1), 107-113. https://doi.org/10.1006/abbi.1993.1261.

2. Greiner, R.; Konietzny, U.; Jany, K. D. Purification and Properties of a Phytase from Rye. Journal of Food Biochemistry 1998, 22 (2), 143-161. https: / /doi.org/10.1111/j.1745-4514.1998.tb00236.x.

3. Greiner, R., Silva, L. G. D., Couri, S. Purification and characterization of an extracellular phytase from Aspergillus niger 11T53A9. Brazilian Journal of Microbiology 2009, 40(4), 795-807.

4. Wyss, M.; Brugger, R.; Kronenberger, A.; Rémy, R.; Fimbel, R.; Oesterhelt, G.; Lehmann, M.; van Loon, A. P. Biochemical Characterization of Fungal Phytases (MyoInositol Hexakisphosphate Phosphohydrolases): Catalytic Properties. Applied and environmental microbiology 1999, 65 (2), 367-373.

5. Konietzny, U.; Greiner, R. Molecular and Catalytic Properties of Phytate-Degrading Enzymes (Phytases). International Journal of Food Science and Technology 2002, 37(7), 791-812. https: //doi.org/10.1046/j.1365-2621.2002.00617.x.

6. Kim, T.; Mullaney, E. J.; Porres, J. M.; Roneker, K. R.; Crowe, S.; Rice, S.; Ko, T.; Ullah, A. H. J.; Daly, C. B.; Welch, R.; Lei X. G. Shifting the PH Profile of Aspergillus niger PhyA Phytase to Match the Stomach PH Enhances Its Effectiveness as an Animal Feed Additive. Applied and Environmental Microbiology 2006, 72 (6), 4397-4403. https: / / doi.org/10.1128/AEM.02612-05.

7. Chen, Q. C.; Li, B. W. Separation of Phytic Acid and Other Related Inositol Phosphates by High-Performance Ion Chromatography and Its Applications. Journal 
of Chromatography A 2003, 1018 (1), 41-52.

https: //doi.org/10.1016/j.chroma.2003.08.040.

8. Casey, A., Walsh, G. Purification and characterization of extracellular phytase from Aspergillus niger ATCC 9142. Bioresource Technology 2003, 86(2), 183-188.

9. George, T. S., Simpson, R. J., Gregory, P. J., Richardson, A. E. Differential interaction of Aspergillus niger and Peniophora lycii phytases with soil particles affects the hydrolysis of inositol phosphates. Soil Biology and Biochemistry 2007, 39(3), 793-803.

10. Sariyska, M. V., Gargova, S. A., Koleva, L. A., Angelov, A. I. Aspergillus niger phytase: purification and characterization. Biotechnology and Biotechnological Equipment 2005, 19(3), 98-105.

11. Stuart, A. (Department of S. at the L. S. of E. Kendall's Advanced Theory of Statistics, 6th ed.; Ord, J. K. (Department of M. S. and S. at P. S. U., Ed.; Wiley: London, 1998; Vol. 